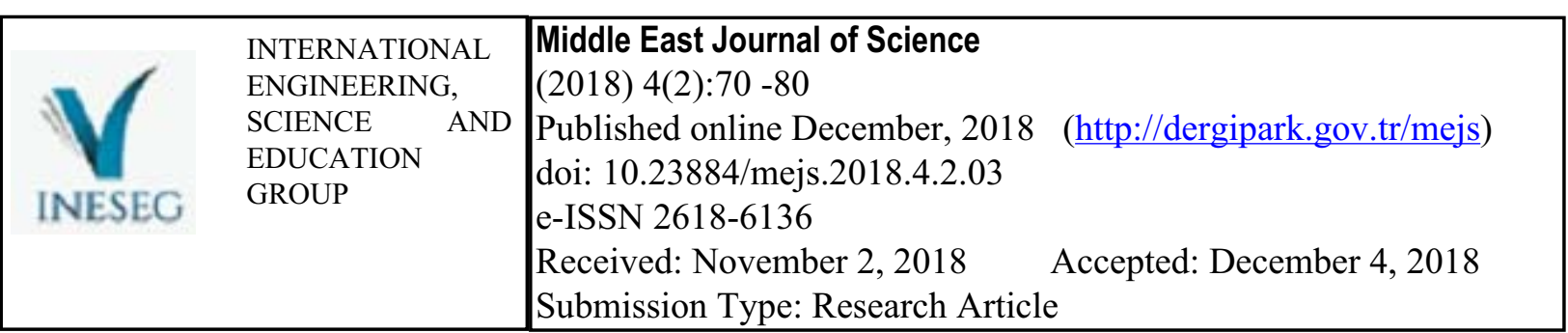

\title{
BLOW UP OF SOLUTIONS FOR A TIMOSHENKO EQUATION WITH DAMPING TERMS
}

\author{
Erhan Pişkin* and Hazal Yüksekkaya \\ Dicle University, Department of Mathematics, Diyarbakır, Turkey \\ ${ }^{*}$ Corresponding author: episkin@dicle.edu.tr
}

\begin{abstract}
In this work, we studied the following equation

$$
u_{t t}+\triangle^{2} u-M\left(\|\nabla u\|^{2}\right) \triangle u-\triangle u_{t}+u_{t}=|u|^{q-1} u
$$

regard to initial and Dirichlet boundary condition. We show that the blow up of solutions with positive and negative initial energy.
\end{abstract}

Keywords: Timoshenko equation, Blow up, Damping term.

Mathematics Subject Classification (2010): 35A01.

\section{Introduction}

In this work, we consider the following Timoshenko equation

$$
\begin{cases}u_{t t}+\triangle^{2} u-M\left(\|\nabla u\|^{2}\right) \triangle u-\triangle u_{t}+u_{t}=|u|^{q-1} u, & (x, t) \in \Omega \times(0, T), \\ u(x, 0)=u_{0}(x), u_{t}(x, 0)=u_{1}(x), & x \in \Omega, \\ u(x, t)=\frac{\partial}{\partial \nu} u(x, t)=0, & x \in \partial \Omega,\end{cases}
$$

where $\Omega$ is a bounded domain of $R^{n}$ having a smooth boundary $\partial \Omega$. Also $q \geq 1$ is real numbers, outer normal is denoted by $\nu$ and $M(s)=1+s^{\gamma}, \gamma \geq 1$.

In the event of $M(s)=1$, without fourth order term $\left(\triangle^{2} u\right)$ and strong damping term $\left(-\triangle u_{t}\right)$ the equation (1) can be recorded in the following form

$$
u_{t t}-\triangle u+u_{t}=|u|^{q-1} u \text {. }
$$

Georgiev and Todorova, Levine, Messaoudi, Vitillaro made further efforts to get the existence and blow up in finite time of solutions for (2). 
In the event of $M(s)=0$ and absent the strong damping term the equation (1) can be typed in the following form

$$
u_{t t}+\triangle^{2} u+u_{t}=|u|^{q-1} u .
$$

Messaoudi [11] researched the local existence and studied blow up of the solution to the equation (3). Wu and Tsai [16] got global existence and made researches about blow up of the solution of the problem (3). Then, blow up of the solution for the problem (3) with positive initial energy was studied by Chen and Zhou [2] .

The problem (1) was researched by Esquivel-Avila [4, 5], he demonstrated blow up, unboundedness, convergence and made researches for global attractor. Pişkin [12] researched the local and global existence, asymptotic behavior also studied about blow up of the solution. Later, Pişkin and Irkıl [13] investigated blow up of the solutions (1) for positive initial energy.

In this paper, we show the blow up of solutions of the problem (1), for positive and negative initial energy.

This work is arranged as the following. In chapter 2, some lemmas and notations are given. In chapter 3, blow up of the solution is discussed.

\subsection{Derivation of the Timoshenko equation}

In this section, we show the derivation of the Timoshenko equation [3, 14].

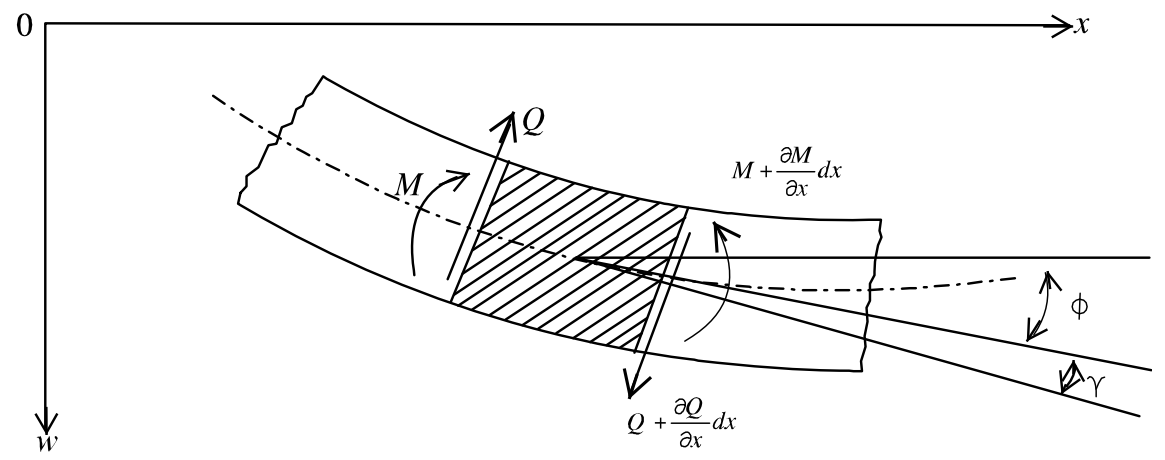

In the foregoing figure, the bending moment is indicated by $M$ and shearing force is indicated by $Q$. Also $\phi$ is the angle of bending and $\gamma$ is the angle of shearing. Deflection is stated by $W$.

For a great number of minuscule deflections

$$
\frac{\partial W}{\partial x}=\phi+\gamma
$$

and by elementary beam theory

$$
\left\{\begin{array}{c}
M=-E I \frac{\partial \phi}{\partial x}, \\
Q=k A G \gamma
\end{array}\right.
$$


Here, flexural rigidity is denoted by $E I ; k$ is a constant related to the form of cross-section of a beam; $A$ is field of cross-section and modulus of rigidity is denoted by $G$.

The movements equations are:

The rotations equation is

$$
-\frac{\partial M}{\partial x} d x+Q d x=\rho I \frac{\partial^{2} \phi}{\partial t^{2}} d x
$$

Here, the density of the material is $\rho$.

In the direction of $W$, the equation for translation is-

$$
\frac{\partial Q}{\partial x} d x=\rho A \frac{\partial^{2} W}{\partial t^{2}} d x
$$

In equation (5), if the account of $Q$ is substituted into equations (6) and (7), we get

$$
\begin{gathered}
-\frac{\partial M}{\partial x}+k A G \gamma=\rho I \frac{\partial^{2} \phi}{\partial t^{2}}, \\
\frac{\partial(k A G \gamma)}{\partial x}=\rho A \frac{\partial^{2} W}{\partial t^{2}} .
\end{gathered}
$$

Substituting for

$$
\gamma=\frac{\partial W}{\partial x}-\phi
$$

in the equation (4) and

$$
M=-E I \frac{\partial \phi}{\partial x}
$$

in the equation (5) into equations (8) and (9), we attain

$$
\begin{gathered}
E I \frac{\partial^{2} \phi}{\partial x^{2}}+k A G\left(\frac{\partial W}{\partial x}-\phi\right)-\rho I \frac{\partial^{2} \phi}{\partial t^{2}}=0, \\
\rho A \frac{\partial^{2} W}{\partial t^{2}}-k A G\left(\frac{\partial^{2} W}{\partial x^{2}}-\frac{\partial \phi}{\partial x}\right)=0 .
\end{gathered}
$$

To eliminate $\phi$ from equations (10) and (11), we rearrange (12) to read

$$
\frac{\partial \phi}{\partial x}=-\frac{\rho A}{k A G} \frac{\partial^{2} W}{\partial t^{2}}+\frac{\partial^{2} W}{\partial x^{2}} .
$$

Now differentiating equation (10) accordinly to $x$ and substituting for $\frac{\partial \phi}{\partial x}$ we attain

$$
\begin{array}{ll} 
& E I \frac{\partial^{2}}{\partial x^{2}}\left[-\frac{\rho A}{k A G} \frac{\partial^{2} W}{\partial t^{2}}+\frac{\partial^{2} W}{\partial x^{2}}\right] \\
+ & k A G\left[\frac{\partial^{2} W}{\partial x^{2}}+\frac{\rho A}{k A G} \frac{\partial^{2} W}{\partial t^{2}}-\frac{\partial^{2} W}{\partial x^{2}}\right] \\
& -\rho I \frac{\partial^{2}}{\partial t^{2}}\left[-\frac{\rho A}{k A G} \frac{\partial^{2} W}{\partial t^{2}}+\frac{\partial^{2} W}{\partial x^{2}}\right] \\
= & 0 .
\end{array}
$$


Simplifying the above expression we obtain

$$
-\frac{E I \rho}{k G} \frac{\partial^{4} W}{\partial x^{2} \partial t^{2}}+E I \frac{\partial^{4} W}{\partial x^{4}}+\rho A \frac{\partial^{2} W}{\partial t^{2}}+\frac{\rho^{2} I}{k G} \frac{\partial^{4} W}{\partial t^{4}}-\rho I \frac{\partial^{4} W}{\partial x^{2} \partial t^{2}}=0,
$$

therefore

$$
E I \frac{\partial^{4} W}{\partial x^{4}}-\rho I\left(1+\frac{E}{k G}\right) \frac{\partial^{4} W}{\partial x^{2} \partial t^{2}}+\rho A \frac{\partial^{2} W}{\partial t^{2}}+\frac{\rho^{2} I}{k G} \frac{\partial^{4} W}{\partial t^{4}}=0 .
$$

This equation is termed the "Timoshenko equation".

Rotatory inertia is symbolized by

$$
-\rho I \frac{\partial^{4} W}{\partial x^{2} \partial t^{2}}
$$

in equation (12) and amendment related to shear by

$$
-\frac{\rho I E}{k G} \frac{\partial^{4} W}{\partial x^{2} \partial t^{2}}+\frac{\rho^{2} I}{k G} \frac{\partial^{4} W}{\partial t^{4}} .
$$

The Euler's equation (13) is got from the Timoshenko equation by sifting the amendments related to both shear and rotatory inertia.

$$
E I \frac{\partial^{4} W}{\partial x^{4}}+\rho A \frac{\partial^{2} W}{\partial t^{2}}=0 .
$$

The Timoshenko beam theory can be thought like a system, such as (10) and (11) or in the one form, as equation (12).

\section{Preliminaries}

In this chapter, we should show some assumptions and lemmas which will be taken advantage of. Where $\|$.$\| and \|.\|_{p}$ indicate the usual $L^{2}(\Omega)$ norm and $L^{p}(\Omega)$ norm, in turn.

Lemma 1 (Sobolev-Poincare inequality) [1]. Let $p$ be a number with $2 \leq p<\infty(n=1,2)$ or $2 \leq p \leq \frac{2 n}{n-2}(n \geq 3)$, and $C_{*}=C_{*}(\Omega, p)$ is a constant, such that

$$
\|u\|_{p} \leq C_{*}\|\nabla u\| \text { for } u \in H_{0}^{1}(\Omega) .
$$

We identify the energy function as follows

$$
\begin{aligned}
E(t)= & \frac{1}{2}\left\|u_{t}\right\|^{2}+\frac{1}{2}\left(\|\nabla u\|^{2}+\|\Delta u\|^{2}\right) \\
& +\frac{1}{2(\gamma+1)}\|\nabla u\|^{2(\gamma+1)}-\frac{1}{q+1}\|u\|_{q+1}^{q+1} .
\end{aligned}
$$

Lemma $2 E(t)$ is a nonincreasing function also $t \geq 0$ and

$$
E^{\prime}(t)=-\left\|u_{t}\right\|^{2}-\left\|\nabla u_{t}\right\|^{2} \leq 0 .
$$


Proof. If we multiply the equation of (1) by $u_{t}$ and integrate over $\Omega$, use integrating by parts, we attain

$$
E(t)-E(0)=-\int_{0}^{t}\left(\left\|u_{\tau}\right\|^{2}+\left\|\nabla u_{\tau}\right\|^{2}\right) d \tau \text { for } t \geq 0
$$

Also, we remark the local existence theorem of problem (1), the proof of it can be present in $[12]$.

Theorem 3 (Local existence). Supposing that $\left(u_{0}, u_{1}\right) \in H_{0}^{2}(\Omega) \times L^{2}(\Omega)$ ensures, after there is an only solution $u$ of (1) satisfying

$$
\begin{gathered}
u \in C\left([0, T) ; H_{0}^{2}(\Omega)\right), \\
u_{t} \in C\left([0, T) ; L^{2}(\Omega)\right) \cap L^{p+1}(\Omega \times(0, T)) .
\end{gathered}
$$

Furthermore, at a minimum one of the following expressions holds:

(i) $T=\infty$,

(ii) $\left\|u_{t}\right\|^{2}+\|\triangle u\|^{2} \longrightarrow \infty$ as $t \longrightarrow T^{-}$.

\section{Blow up of solutions}

In this chapter, we work away the blow up of the solution for the problem (1). We should denote the following two lemmas, which will be taken advantage of then.

Lemma 4 [9]. Let $\delta>0$ and $B(t) \in C^{2}(0, \infty)$ be a nonnegative function satisfying

$$
B^{\prime \prime}(t)-4(\delta+1) B^{\prime}(t)+4(\delta+1) B(t) \geq 0 .
$$

If

$$
B^{\prime}(0)>r_{2} B(0)+K_{0},
$$

with $r_{2}=2(\delta+1)-2 \sqrt{(\delta+1) \delta}$, then $B^{\prime}(t)>K_{0}$ for $t>0$, here $K_{0}$ is a constant.

Lemma 5 [9]. If $H(t)$ is a nonincreasing function on $\left[t_{0}, \infty\right)$ and satisfies the differential inequality

$$
\left[H^{\prime}(t)\right]^{2} \geq a+b[H(t)]^{2+\frac{1}{\delta}}, \text { for } t \geq t_{0},
$$

where $a>0, b \in R$, then there exists a finite time $T^{*}$ such that

$$
\lim _{t \longrightarrow T^{*-}} H(t)=0
$$


Upper bounds for $T^{*}$ are estimated as follows:

(i) If $b<0$ and $H\left(t_{0}\right)<\min \left\{1, \sqrt{-\frac{a}{b}}\right\}$ then

$$
T^{*} \leq t_{0}+\frac{1}{\sqrt{-b}} \ln \frac{\sqrt{-\frac{a}{b}}}{\sqrt{-\frac{a}{b}}-H\left(t_{0}\right)} .
$$

(ii) If $b=0$, then

$$
T^{*} \leq t_{0}+\frac{H\left(t_{0}\right)}{\sqrt{a}}
$$

(iii) If $b>0$, then

$$
T^{*} \leq \frac{H\left(t_{0}\right)}{\sqrt{a}} \text { or } T^{*} \leq t_{0}+2^{\frac{3 \delta+1}{2 \delta}} \frac{\delta c}{\sqrt{a}}\left[1-\left(1+c H\left(t_{0}\right)\right)^{-\frac{1}{2 \delta}}\right],
$$

where $c=\left(\frac{a}{b}\right)^{\frac{\delta}{2 \delta+1}}$.

Definition 6 A solution $u$ of (1) is termed blow up if there is a finite time $T^{*}$ such that

$$
\lim _{t \longrightarrow T^{*-}}\left[\int_{\Omega} u^{2} d x+\int_{0}^{t} \int_{\Omega}\left(u^{2}+|\nabla u|^{2}\right) d x d \tau\right]=\infty .
$$

Let

$$
a(t)=\int_{\Omega} u^{2} d x+\int_{0}^{t} \int_{\Omega}\left(u^{2}+|\nabla u|^{2}\right) d x d \tau, \text { for } t \geq 0 .
$$

Lemma 7 Assume $\frac{q-1}{4} \geq \delta \geq \frac{\gamma}{2}$, and that $\gamma \geq 0$, then we have

$$
a^{\prime \prime}(t) \geq 4(\delta+1) \int_{\Omega} u_{t}^{2} d x-4(2 \delta+1) E(0)+4(2 \delta+1) \int_{0}^{t}\left(\left\|u_{\tau}\right\|^{2}+\left\|\nabla u_{\tau}\right\|^{2}\right) d \tau .
$$

Proof. By differentiating (21) according to t, we have

$$
\begin{gathered}
a^{\prime}(t)=2 \int_{\Omega} u u_{t} d x+\|u\|^{2}+\|\nabla u\|^{2} \\
a^{\prime \prime}(t)=2 \int_{\Omega} u_{t}^{2} d x+2 \int_{\Omega} u u_{t t} d x+2 \int_{\Omega} u u_{t} d x+2 \int_{\Omega} \nabla u \nabla u_{t} d x \\
=2\left(\left\|u_{t}\right\|^{2}+\|u\|_{q+1}^{q+1}\right)-2\left(\|\nabla u\|^{2}+\|\nabla u\|^{2(\gamma+1)}+\|\Delta u\|^{2}\right) .
\end{gathered}
$$

Then from (1) and (24), we have

$$
\begin{aligned}
a^{\prime \prime}(t)= & 4(\delta+1) \int_{\Omega} u_{t}^{2} d x-4(2 \delta+1) E(0) \\
& +4 \delta\left(\|\nabla u\|^{2}+\|\Delta u\|^{2}\right)+\left(\frac{4 \delta+2}{\gamma+1}-2\right)\|\nabla u\|^{2(\gamma+1)} \\
& +\left(2-\frac{4(2 \delta+1)}{q+1}\right)\|u\|_{q+1}^{q+1}+4(2 \delta+1) \int_{0}^{t}\left(\left\|u_{\tau}\right\|^{2}+\left\|\nabla u_{\tau}\right\|^{2}\right) d \tau .
\end{aligned}
$$

Since $\frac{q-1}{4} \geq \delta \geq \frac{\gamma}{2}$, we obtain (22). 
Lemma 8 Assume $\frac{q-1}{4} \geq \delta \geq \frac{\gamma}{2}, \gamma \geq 0$ and one of the following expressions are satisfied

(i) $E(0)<0$ and $\int_{\Omega} u_{0} u_{1} d x>0$,

(ii) $E(0)=0$ and $\int_{\Omega} u_{0} u_{1} d x>0$,

(iii) $E(0)>0$ and

$$
a^{\prime}(0)>r_{2}\left[a(0)+\frac{K_{1}}{4(\delta+1)}\right]+\left\|u_{0}\right\|^{2}+\left\|\nabla u_{0}\right\|^{2}
$$

holds.

Then $a^{\prime}(t)>\left\|u_{0}\right\|^{2}+\left\|\nabla u_{0}\right\|^{2}$ for $t>t^{*}$, where $t_{0}=t^{*}$ is given by (26) in case (i) and $t_{0}=0$ in cases (ii) and (iii).

Where $K_{1}$ and $t^{*}$ are defined in (30) and (26), in turn.

Proof. (i) If $E(0)<0$, then by $(22)$, we attain

$$
a^{\prime}(t) \geq 2 \int_{\Omega} u_{0} u_{1} d x+\left\|u_{0}\right\|^{2}+\left\|\nabla u_{0}\right\|^{2}-4(2 \delta+1) E(0) t, \quad t \geq 0
$$

Thereby we obtain $a^{\prime}(t)>\left\|u_{0}\right\|^{2}+\left\|\nabla u_{0}\right\|^{2}$ for $t>t^{*}$, where

$$
t^{*}=\max \left\{\frac{a^{\prime}(0)-\left(\left\|u_{0}\right\|^{2}+\left\|\nabla u_{0}\right\|^{2}\right)}{4(2 \delta+1) E(0)}, 0\right\} .
$$

(ii) If $E(0)=0$ and $\int_{\Omega} u_{0} u_{1} d x>0$, then $a^{\prime \prime}(t) \geq 0$ for $t \geq 0$. We have $a^{\prime}(t)>\left\|u_{0}\right\|^{2}+$ $\left\|\nabla u_{0}\right\|^{2}, t \geq 0$.

(iii) If $E(0)>0$, firstly, we write down that

$$
2 \int_{0}^{t} \int_{\Omega} u u_{t} d x d \tau=\|u\|^{2}-\left\|u_{0}\right\|^{2} .
$$

Utilising Hölder inequality and Young inequality, we obtain

$$
\|u\|^{2} \leq\left\|u_{0}\right\|^{2}+\int_{0}^{t}\|u\|^{2} d \tau+\int_{0}^{t}\left\|u_{\tau}\right\|^{2} d \tau
$$

From (21), (23) and (28), we attain

$$
a^{\prime}(t) \leq a(t)+\left\|u_{0}\right\|^{2}+\left\|u_{t}\right\|^{2}+\int_{0}^{t} \int_{\Omega}\left(u_{\tau}^{2}+|\nabla u|^{2}\right) d x d \tau
$$

Hence, by (22) and (29), we get

$$
a^{\prime \prime}(t)-4(\delta+1) a^{\prime}(t)+4(\delta+1) a(t)+K_{1} \geq 0
$$

where

$$
\begin{aligned}
K_{1}= & 4(2 \delta+1) E(0)+4(\delta+1) \int_{\Omega} u_{0}^{2} d x \\
& +4(\delta+1) \int_{\Omega}|\nabla u|^{2} d x-4 \delta \int_{0}^{t}\left(\left\|u_{\tau}\right\|^{2}+\left\|\nabla u_{\tau}\right\|^{2}\right) d \tau
\end{aligned}
$$


Let

$$
b(t)=a(t)+\frac{K_{1}}{4(\delta+1)}, \quad t>0 .
$$

After $b(t)$ provides Lemma 4 . As a result, we obtain by $(25) a^{\prime}(t)>\left\|u_{0}\right\|^{2}+\left\|\nabla u_{0}\right\|^{2}, t>0$, where $r_{2}$ is given in Lemma 4 .

Theorem 9 Assume $\frac{q-1}{4} \geq \delta \geq \frac{\gamma}{2}, \gamma \geq 0$ and one of the following expressions are satisfied

(i) $E(0)<0$ and $\int_{\Omega} u_{0} u_{1} d x>0$,

(ii) $E(0)=0$ and $\int_{\Omega} u_{0} u_{1} d x>0$,

(iii) $0<E(0)<\frac{\left(a^{\prime}\left(t_{0}\right)-\left(\left\|u_{0}\right\|^{2}+\left\|\nabla u_{0}\right\|^{2}\right)\right)^{2}}{8\left[a\left(t_{0}\right)+\left(T_{1}-t_{0}\right)\left(\left\|u_{0}\right\|^{2}+\left\|\nabla u_{0}\right\|^{2}\right)\right]}$ and (25) holds.

After the solution $u$ blow up in finite time $T^{*}$ in the case of (30). In case (i),

$$
T^{*} \leq t_{0}-\frac{H\left(t_{0}\right)}{H^{\prime}\left(t_{0}\right)}
$$

Moreover, if $H\left(t_{0}\right)<\min \left\{1, \sqrt{-\frac{a}{b}}\right\}$, we get

$$
T^{*} \leq t_{0}+\frac{1}{\sqrt{-b}} \ln \frac{\sqrt{-\frac{a}{b}}}{\sqrt{-\frac{a}{b}}-H\left(t_{0}\right)},
$$

where

$$
\begin{gathered}
a=\delta^{2} H^{2+\frac{2}{\delta}}\left(t_{0}\right)\left[\left(a^{\prime}\left(t_{0}\right)-\left\|u_{0}\right\|^{2}\right)^{2}-8 E(0) H^{-\frac{1}{\delta}}\left(t_{0}\right)\right]>0, \\
b=8 \delta^{2} E(0) .
\end{gathered}
$$

In case (ii),

$$
T^{*} \leq t_{0}-\frac{H\left(t_{0}\right)}{H^{\prime}\left(t_{0}\right)}
$$

In case (iii),

$$
T^{*} \leq \frac{H\left(t_{0}\right)}{\sqrt{a}} \text { or } T^{*} \leq t_{0}+2^{\frac{3 \delta+1}{2 \delta}}\left(\frac{a}{b}\right)^{2+\frac{1}{\delta}} \frac{\delta}{\sqrt{a}}\left\{1-\left[1+\left(\frac{a}{b}\right)^{2+\frac{1}{\delta}} H\left(t_{0}\right)\right]^{-\frac{1}{2 \delta}}\right\},
$$

where $a$ and $b$ are given (33), (34).

Proof. Let

$$
H(t)=\left[a(t)+\left(T_{1}-t\right)\left(\left\|u_{0}\right\|^{2}+\left\|\nabla u_{0}\right\|^{2}\right)\right]^{-\delta}, \text { for } t \in\left[0, T_{1}\right],
$$

where $T_{1}>0$ is a specific constant that will be indicated then. Later, we obtain

$$
\begin{gathered}
H^{\prime}(t)=-\delta\left[a(t)+\left(T_{1}-t\right)\left(\left\|u_{0}\right\|^{2}+\left\|\nabla u_{0}\right\|^{2}\right)\right]^{-\delta-1}\left[a^{\prime}(t)-\left(\left\|u_{0}\right\|^{2}+\left\|\nabla u_{0}\right\|^{2}\right)\right] \\
=-\delta H^{1+\frac{1}{\delta}}(t)\left[a^{\prime}(t)-\left(\left\|u_{0}\right\|^{2}+\left\|\nabla u_{0}\right\|^{2}\right)\right] \\
H^{\prime \prime}(t)=-\delta H^{1+\frac{2}{\delta}}(t) a^{\prime \prime}(t)\left[a(t)+\left(T_{1}-t\right)\left(\left\|u_{0}\right\|^{2}+\left\|\nabla u_{0}\right\|^{2}\right)\right] \\
+\delta H^{1+\frac{2}{\delta}}(t)(1+\delta)\left[a^{\prime}(t)-\left(\left\|u_{0}\right\|^{2}+\left\|\nabla u_{0}\right\|^{2}\right)\right]^{2}
\end{gathered}
$$


and

$$
H^{\prime \prime}(t)=-\delta H^{1+\frac{2}{\delta}}(t) V(t)
$$

where

$$
V(t)=a^{\prime \prime}(t)\left[a(t)+\left(T_{1}-t\right)\left(\left\|u_{0}\right\|^{2}+\left\|\nabla u_{0}\right\|^{2}\right)\right]-(1+\delta)\left[a^{\prime}(t)-\left(\left\|u_{0}\right\|^{2}+\left\|\nabla u_{0}\right\|^{2}\right)\right]^{2} .
$$

For simplicity of calculation, we define

$$
\begin{gathered}
P_{u}=\int_{\Omega} u^{2} d x, \quad R_{u}=\int_{\Omega} u_{t}^{2} d x, \\
Q_{u}=\int_{0}^{t}\|u\|^{2} d t, \quad S_{u}=\int_{0}^{t}\left\|u_{t}\right\|^{2} d t, \\
M_{u}=\int_{0}^{t}\|\nabla u\|^{2} d \tau, \quad N_{u}=\int_{0}^{t}\left\|\nabla u_{\tau}\right\|^{2} d \tau .
\end{gathered}
$$

From (23), (27) and Hölder inequality, we get

$$
\begin{aligned}
a^{\prime}(t) & =2 \int_{\Omega} u u_{t} d x+\left\|u_{0}\right\|^{2}+\|\nabla u\|^{2}+2 \int_{0}^{t} \int_{\Omega} u u_{t} d x d t \\
& \leq 2\left(\sqrt{R_{u} P_{u}}+\sqrt{Q_{u} S_{u}}\right)+\left\|u_{0}\right\|^{2}+\|\nabla u\|^{2} .
\end{aligned}
$$

If case (i) or (ii) holds, from (22) we get

$$
a^{\prime \prime}(t) \geq(-4-8 \delta) E(0)+4(1+\delta)\left(R_{u}+S_{u}+N_{u}\right) .
$$

Thus, from (41)-(43) and (37), we attain

$$
\begin{aligned}
V(t) \geq & {\left[(-4-8 \delta) E(0)+4(1+\delta)\left(R_{u}+S_{u}+N_{u}\right)\right] H^{-\frac{1}{\delta}}(t) } \\
& -4(1+\delta)\left(\sqrt{R_{u} P_{u}}+\sqrt{Q_{u} S_{u}}+\sqrt{M_{u} N_{u}}\right)^{2}
\end{aligned}
$$

From (21),

$$
\begin{aligned}
a(t) & =\int_{\Omega} u^{2} d x+\int_{0}^{t} \int_{\Omega}\left(u^{2}+|\nabla u|^{2}\right) d x d \tau \\
& =P_{u}+Q_{u}+M_{u}
\end{aligned}
$$

and (37), we get

$V(t) \geq(-4-8 \delta) E(0) H^{-\frac{1}{\delta}}(t)+4(1+\delta)\left[\left(R_{u}+S_{u}+N_{u}\right)\left(T_{1}-t\right)\left(\left\|u_{0}\right\|^{2}+\left\|\nabla u_{0}\right\|^{2}\right)+\Theta(t)\right]$

where

$$
\Theta(t)=\left(R_{u}+S_{u}+N_{u}\right)\left(P_{u}+Q_{u}+M_{u}\right)-\left(\sqrt{R_{u} P_{u}}+\sqrt{Q_{u} S_{u}}+\sqrt{M_{u} N_{u}}\right)^{2} .
$$

Utilising the Schwarz inequality, and $\Theta(t)$ being nonnegative, we get

$$
V(t) \geq(-4-8 \delta) E(0) H^{-\frac{1}{\delta}}(t), t \geq t_{0}
$$


Thus, from (40) and (44), we obtain

$$
H^{\prime \prime}(t) \leq 4 \delta(1+2 \delta) E(0) H^{1+\frac{1}{\delta}}(t), t \geq t_{0}
$$

From Lemma 8, we recognise that $H^{\prime}(t)<0$ for $t \geq t_{0}$. Multiplying (45) by $H^{\prime}(t)$ and integrating it from $t_{0}$ to $t$, we obtain

$$
H^{\prime 2}(t) \geq a+b H^{2+\frac{1}{\delta}}(t)
$$

for $t \geq t_{0}$, we can see $a, b$ are described in (33) and (34) in turn.

If case (iii) holds, similar to the steps of case (i), we obtain $a>0$ if and only if

$$
E(0)<\frac{\left(a^{\prime}\left(t_{0}\right)-\left(\left\|u_{0}\right\|^{2}+\left\|\nabla u_{0}\right\|^{2}\right)\right)^{2}}{8\left[a\left(t_{0}\right)+\left(T_{1}-t_{0}\right)\left(\left\|u_{0}\right\|^{2}+\left\|\nabla u_{0}\right\|^{2}\right)\right]} .
$$

After, from Lemma 5, there is a finite time $T^{*}$ such that $\lim _{t \rightarrow T^{*-}} H(t)=0$ and upper bound of $T^{*}$ is estimated for the sign of $E(0)$. This implies that (20) provides.

\section{References}

[1] Adams, R.A., Fournier, J.J.F., Sobolev Spaces, Academic Press, New York, 2003.

[2] Chen, W., Zhou, Y., Global nonexistence for a semilinear Petrovsky equation, Nonlinear Anal., 70, 3203-3208, 2009.

[3] Doshi,C., On the Analysis of the Timoshenko Beam Theory with and without internal damping, Thesis, Rochester Institute of Technology, 1979.

[4] Esquivel-Avila, J.A., Dynamic analysis of a nonlinear Timoshenko equation, Abstract and Applied Analysis, 2011, 1-36, 2010.

[5] Esquivel-Avila, J.A., Global attractor for a nonlinear Timoshenko equation with source terms, Mathematical Sciences, 1-8, 2013.

[6] Georgiev, V., Todorova, G., Existence of a solution of the wave equation with nonlinear damping and source term, Journal of Differential Equations, 109, 295-308, 1994.

[7] Levine, H.A., Instability and nonexistence of global solutions to nonlinear wave equations of the form $P u_{t t}=-A u+F(u)$, Trans. Amer. Math. Soc., 192, 1-21, 1974.

[8] Levine, H.A., Some additional remarks on the nonexistence of global solutions to nonlinear wave equations, SIAM Journal on Applied Mathematics, 5, 138-146, 1974. 
[9] Li, M.R., Tsai, L.Y., Existence and nonexistence of global solutions of some system of semilinear wave equations, Nonlinear Anal., 54 (8), 1397-1415, 2003.

[10] Messaoudi, S.A., Blow up in a nonlinearly damped wave equation, Mathematische Nachrichten, 231, 105-111, 2001.

[11] Messaoudi, S.A., Global existence and nonexistence in a system of Petrovsky, J. Math. Anal. Appl., 265(2), 296-308, 2002.

[12] Pişkin, E., Existence, decay and blow up of solutions for the extensible beam equation with nonlinear damping and source terms, Open Math., 13, 408-420, 2015.

[13] Pişkin E., Irkıl, N., Blow up of Positive Initial-Energy Solutions for the Extensible Beam Equation with Nonlinear Damping and Source terms, Ser. Math. Inform., 31(3), 645-654, 2016.

[14] Timoshenko, S.P., On the Correction for Shear of the Differential Equation for Transverse Vibrations of Prismatic Bars, Philosophical Magazine and Journal of Science, 6(41), 744$746,1921$.

[15] Vitillaro, E., Global existence theorems for a class of evolution equations with dissipation, Arch. Rational Mech. Anal., 149, 155-182, 1999.

[16] Wu, S.T., Tsai, L.Y., On global solutions and blow-up of solutions for a nonlinearly damped Petrovsky system, Taiwanese J. Math., 13 (2A), 545-558, 2009. 